\title{
How Useful is Extravascular Lung Water Measurement in Managing Lung Injury in Intensive Care Unit?
}

\author{
Anirban Bhattacharjee, Debasis Pradhan, Prithwis Bhattacharyya, Samarjit Dey, Daniala Chhunthang'1, Akash Handique', Angkita Barman, Mohd Yunus \\ Departments of Anaesthesiology and Critical Care and ${ }^{~}$ Radiology and Imaging, NEIGRIHMS, Shillong, Meghalaya, India
}

\section{Abstract}

Context: The primary goal of septic shock management is optimization of organ perfusion, often at the risk of overloading the interstitium and causing pulmonary edema. The conventionally used end points of resuscitation do not generally include volumetric parameters such as extravascular lung water index (EVLWI) and pulmonary vascular permeability index (PVPI). Aims: This study aimed to assess the prognostic value of EVLWI and PVPI by calculating their correlation with the severity of lung injury. Settings and Design: This prospective observational study included twenty mechanically ventilated critically ill patients with Acute Physiology and Chronic Health Evaluation score (APACHE II) $>20$. Subjects and Methods: EVLWI and PVPI were measured using transpulmonary thermodilution, and simultaneously, $\mathrm{PaO}_{2}: \mathrm{FiO}_{2}$ ratio, alveolar-arterial gradient of oxygen $\left(\mathrm{AaDO}_{2}\right)$, and chest radiograph scores from two radiologists were obtained. Statistical Analysis: The correlation of EVLWI and PVPI with chest radiograph scores, $\mathrm{PaO}_{2}: \mathrm{FiO}_{2}$ ratio, and $\mathrm{AaDO}_{2}$ were calculated. The inter-observer agreement between the two radiologists was tested using kappa test. Results: EVLWI and PVPI correlated modestly with $\mathrm{PaO}_{2}: \mathrm{FiO}_{2}(r=-0.32, P=0.0004 ; r=-0.39, P=0.0001)$. There was a better correlation of EVLWI and $\mathrm{PVPI}$ with $\mathrm{PaO}_{2}:$ FiO ${ }_{2}$ ratio $(r=-0.71, P<0.0001 ; r=-0.58, P=0.0001)$ in the acute respiratory distress syndrome (ARDS) subgroup. The EVLWI values correlated significantly with corresponding chest radiograph scores $(r=0.71, P<0.0001$ for observer 1 and $r=0.68, P<0.0001$ for observer 2$)$. Conclusions: EVLWI and PVPI may have a prognostic significance in the assessment of lung injury in septic shock patients with ARDS. Further research is required to reveal the usefulness of EVLWI as an end point of fluid resuscitation in the management of septic shock with ARDS.

Keywords: Acute respiratory distress syndrome, extravascular lung water, septic shock, thermodilution

\section{INTRODUCTION}

Extravascular lung water (EVLW) is the water within the lungs outside the pulmonary circulation and this amounts to the sum of interstitial, intracellular, alveolar, and lymphatic fluid excluding pleural effusions. ${ }^{[1]}$ Pulmonary vascular permeability index (PVPI) is the ratio of EVLW and pulmonary blood volume (PBV), which is an indirect reflection of integrity of pulmonary capillary barrier. Two commercially available transpulmonary thermodilution (TPTD) devices (PICCO2, Maquet $^{\circledR}$, Munich, Germany, and VolumeView/EV $1000^{\circledR}$, Edwards Life Sciences, Irvine, CA, USA) are being used to measure EVLW index (EVLWI) and PVPI in addition to other hemodynamic parameters. ${ }^{[2]}$ While EVLW is the absolute amount of lung water measured, EVLWI is the ratio of EVLW with the patient's actual or predicted body weight to nullify the variation due to patient's anthropometric measures.

\begin{tabular}{|l|l|}
\hline \multicolumn{2}{|c|}{ Access this article online } \\
\hline Quick Response Code: & Website: \\
\hline & www.ijccm.org \\
\cline { 2 - 2 } & \\
\hline
\end{tabular}

The primary goal of septic shock management is maintenance of organ perfusion. ${ }^{[3]}$ In our endeavor to perfuse organs in the face of systemic vasodilatation and "leaky" capillaries, we can cause pulmonary edema and affect oxygenation, thereby compromising the delivery of oxygen. ${ }^{[4]}$ Again, appropriate fluid resuscitation protocol cannot be clearly defined without the knowledge of pulmonary vascular permeability. The conventional end points of resuscitation seldom include volumetric parameters. Parameters of fluid responsiveness cannot always guide us about the adequacy of fluid administration and adverse effects of overresuscitation.

Address for correspondence: Prof. Prithwis Bhattacharyya, Department of Anaesthesiology and Critical Care, NEIGRIHMS, 105, Mawdiangdiang, East Khasi Hills, Shillong - 793 018, Meghalaya, India. E-mail: prithwisbhat123@gmail.com

This is an open access article distributed under the terms of the Creative Commons Attribution-NonCommercial-ShareAlike 3.0 License, which allows others to remix, tweak, and build upon the work non-commercially, as long as the author is credited and the new creations are licensed under the identical terms.

For reprints contact: reprints@medknow.com

How to cite this article: Bhattacharjee A, Pradhan D, Bhattacharyya $\mathrm{P}$, Dey S, Chhunthang D, Handique A, et al. How useful is extravascular lung water measurement in managing lung injury in intensive care unit?. Indian J Crit Care Med 2017;21:494-9. 
Based on EVLW and PVPI, we can decide whether to stop fluid administration with or without the need of fluid removal. In the recent years, many studies in the field of critical care, sepsis, and ARDS have focused on the validation of EVLW measurement and its ability to characterize lung edema, prognosticate critically ill patients, and facilitate lung-targeted treatments and fluid management strategies. ${ }^{[2]}$ We conducted a prospective observational study in the Intensive Care Unit (ICU) of our institute, with an aim to assess the correlation between EVLWI and PVPI with the severity of lung injury in patients with septic shock.

\section{Subjects and Methods}

This prospective observational study was conducted in the ICU of our institute after obtaining ethical clearance from the Institutional Ethics Committee during 2014-2016. Following written informed consent, twenty critically ill patients between 18 and 60 years of age with an admission diagnosis of septic shock with or without ARDS with Acute Physiology and Chronic Health Evaluation II $\geq 20$ requiring mechanical ventilation were included in this study. The exclusion criteria included pregnant patients, patients with coagulopathy (international normalized ratio $>1.5$ or platelet count $<100,000$ per cubic millimeters or both), history of pneumonectomy/lobectomy, peripheral arterial disease, contraindication for femoral artery catheterization, patients with a diagnosis of deep vein thrombosis, and pulmonary embolism. None of our patients needed extracorporeal membrane oxygenation support.

EVLW indexed to the predicted body weight, EVLWI, was measured by injecting $20 \mathrm{ml}$ of ice-cold saline through the central venous catheter, through thermistor manifold three times, and the average of the three readings was noted. For this purpose, VolumeView ${ }^{\circledR}$ and EV $1000^{\circledR}$ Clinical Platform (Edwards Life Sciences, Irvine, California, USA) were used. Predicted body weight (in kilograms) was calculated as 0.91 (height in centimeters - 152.4) +50 for males or 0.91 (height in centimeters - 152.4) +45.5 for females. Central venous catheters were inserted through the right internal jugular vein, and catheter tip position in the lower part of superior vena cava was confirmed by chest radiograph (above carina and below sternoclavicular junction). The anteroposterior chest radiographs were obtained along with the EVLW measurements. The radiographic exposure time was minimized to decrease motion artifacts, due to respiratory and cardiac motions. Two radiologists, who were blinded to the volumetric and oxygenation parameters, interpreted each of the radiographs. Each lung was divided into three zones - upper, lower, and perihilar zones. Each of the six zones was given a score from 0 to 65 as follows: 0 - normal, 10 - mild pulmonary vascular congestion, 20 - moderate pulmonary vascular congestion, 30 - severe pulmonary vascular congestion, 40 - interstitial edema without septal lines, 45 - interstitial edema with septal lines, 50 - mixed interstitial and alveolar edema, sparing some areas, 55 - mixed interstitial and alveolar edema, involving the entire lung zone, 60 - alveolar edema with sparing, and 65 - alveolar edema involving the entire lung zone. Summations of scores from the six zones, ranging from 0 to 390 , were the final chest radiograph scores. ${ }^{[5]}$

Simultaneously with EVLWI measurements, $\mathrm{PaO}_{2}: \mathrm{FiO}_{2}$ and alveolar-arterial gradient of oxygen $\left(\mathrm{AaDO}_{2}\right)$ were also recorded. $\mathrm{AaDO}_{2}$ was derived using alveolar gas equation $\left(\mathrm{AaDO}_{2}=\left[\mathrm{FiO}_{2} *\left[\mathrm{P}_{\mathrm{atm}}-\mathrm{P}_{\mathrm{H} 2 \mathrm{O}}\right]-\mathrm{PaCO}_{2} / \mathrm{R}\right]-\mathrm{PaO}_{2}\right.$, $\mathrm{P}_{\text {atm }}$ - atmospheric pressure, $760 \mathrm{mmHg}, \mathrm{P}_{\mathrm{H} 2 \mathrm{O}}$ - saturated water vapor pressure, $47 \mathrm{mmHg}, \mathrm{R}$ - respiratory quotient, $\mathrm{PaO}_{2}$ and $\mathrm{PaCO}_{2}$ - partial pressures of arterial oxygen and carbon dioxide as measured by arterial blood gas) while taking respiratory quotient as 0.8 for all measurements. EVLWI, PVPI, $\mathrm{PaO}_{2}: \mathrm{FiO}_{2}$, and $\mathrm{AaDO}_{2}$ were measured at least twice a day, and chest radiographs were obtained along with EVLWI measurements when clinically indicated. A total of 117 sets of EVLWI values with corresponding $\mathrm{PaO}_{2}: \mathrm{FiO}_{2}$ and $\mathrm{AaDO}_{2}$ readings were obtained for correlation. After rejecting three chest radiographs due to presence of pleural effusion, 64 chest radiographs were scored by the two radiologists and correlated with the corresponding EVLWI readings. Ninety-nine readings of PVPI were recorded and correlated with EVLWI, chest radiograph scores, $\mathrm{PaO}_{2}: \mathrm{FiO}_{2}$ ratio, and $\mathrm{AaDO}_{2}$.

\section{Statistical analysis}

In this study, we correlated between EVLWI and PVPI with chest radiograph scores and oxygenation parameters. Patients were grouped into ARDS and non-ARDS for subgroup analysis. Baseline parameters were described as mean $\pm 2 *$ standard deviation or median (interquartile range). Normality of data was checked with D'Agostino-Pearson test. Correlation between variables was tested by Pearson's coefficient of correlation and graphed as scatter plots. Inter-observer agreement for the two chest radiograph scores was measured by Cohen's kappa test. The significance of each of the correlation coefficients was tested with Student's $t$-test, and $P<0.05$ was considered the cutoff for statistical significance. The statistical analysis was done using the statistical software MedCalc ${ }^{\circledR}$ (v. 12.5.0.0) (Ostend, Belgium).

\section{RESULTS}

This study included twenty patients whose baseline parameters are summarized in Table 1. All patients were mechanically ventilated with lung protective ventilation with median baseline positive end-expiratory pressure of $5 \mathrm{cmH}_{2} \mathrm{O}$. Baseline parameters were found to be normally distributed $(P>0.05)$, but the distributions of the measurements used for correlation were not normal. Mean baseline EVLWI and PVPI were higher in ARDS patients, but the difference was not statistically significant $(P>0.05)$. Fifteen patients died during their ICU stay, four patients were successfully treated and shifted out to the ward. There was no statistically significant difference in mean EVLWI $(P=0.8581)$ and PVPI $(P=0.5744)$ between the two groups. One patient took leave against medical advice and was not included in any of the outcome groups. 
The chest radiograph scores from both radiologists strongly correlated with EVLWI $(r=0.71$ and 0.68 for observers 1 and 2, respectively, $P<0.0001$ for both observers) [Figure 1 and Table 2]. A moderate correlation between chest radiograph scores and PVPI was obtained $(r=0.60$ and 0.59 for observers 1 and 2, respectively, $P<0.0001$ for both observers) [Figure 1 and Table 2]. The chest radiograph scores from the two independent observers correlated strongly with each other $(r=0.78, P<0.0001)$. There was a good inter-observer agreement between the two radiologists with a kappa value of 0.77 (95\% confidence interval: $0.67-0.88)$. A moderately negative correlation was found between EVLWI and $\mathrm{PaO}_{2}: \mathrm{FiO}_{2}$ ratio $(r=-0.32, P=0.0004)$ [Figure 1 and Table 2]. The EVLWI values correlated moderately with $\mathrm{AaDO}_{2}$

\begin{tabular}{|c|c|}
\hline Baseline characteristics & $n=20$ \\
\hline Age & $40(29-50)$ \\
\hline Male sex & $11(55 \%)$ \\
\hline Medical vs post surgical patients & 17 vs 3 \\
\hline ARDS & $9(45 \%)$ \\
\hline Baseline CI (ml/m²) & $4.02 \pm 1.47$ \\
\hline Baseline SVRI (dyne-s-m²/cm5) & $1555.25 \pm 778.98$ \\
\hline Baseline EVLWI (ml/kg) & $13.2(7.45-17.45)$ \\
\hline Baseline PVPI & $3.52(2.7996-4.2404)$ \\
\hline Baseline GEDI (ml/ $\left./ \mathrm{m}^{2}\right)$ & $525.55 \pm 158.09$ \\
\hline APACHE II score & $20.5(20-23.5)$ \\
\hline SOFA score & $11(9-12)$ \\
\hline MODS score & $8(6-10)$ \\
\hline $\mathrm{PaO}_{2}: \mathrm{FiO}_{2}$ ratio & $185.5(100-262.5)$ \\
\hline $\mathrm{AaDO}_{2}$ & $158.2(121.15-364.45)$ \\
\hline Chest Radiograph score (Observer 1) & $180(121-244)$ \\
\hline Chest Radiograph score (Observer 2) & $120(23-228)$ \\
\hline Thoracic Fluid Content & $41(28-47)$ \\
\hline Mechanical ventilation requirement & $20(100 \%)$ \\
\hline Baseline $\mathrm{V}_{\mathrm{T}}(\mathrm{ml} / \mathrm{kg})$ & $8(6-8)$ \\
\hline Baseline PEEP $\left(\mathrm{cm} \mathrm{H}_{2} \mathrm{O}\right)$ & $5(5-7)$ \\
\hline \multicolumn{2}{|c|}{$\begin{array}{l}\text { Data are expressed as Mean } \pm \text { Standarad Deviation, or, Median } \\
\text { (Interquartile range) or specified otherwise. EVLWI: Extravascular lung } \\
\text { water index; APACHE: Acute Physiology and Chronic Health Evaluation; } \\
\text { SOFA: Sequential Organ Failure Assessment score; MODS: Multiple } \\
\text { Organ Dysfunction Score; PEEP: Positive end-expiratoty pressure; } \\
V_{\mathrm{T}} \text { : Tidal volume }\end{array}$} \\
\hline
\end{tabular}

values $(r=0.29, P=0.0014)$ [Figure 1 and Table 2]. There was a moderate correlation between PVPI and $\mathrm{PaO}_{2}: \mathrm{FiO}_{2}(r=-0.39, P=0.0001)$ and $\mathrm{AaDO}_{2}(r=0.32$, $P=0.0013$ ) [Figure 1 and Table 2]. However, there was a good correlation of EVLWI with $\mathrm{PaO}_{2}: \mathrm{FiO}_{2}(r=-0.71, P<0.0001)$ and $\mathrm{AaDO}_{2}(r=0.66, P=0.0001)$ among ARDS patients [Table 3]. PVPI among ARDS patients was better correlated with $\mathrm{PaO}_{2}: \mathrm{FiO}_{2}(r=-0.58, P=0.0001)$ and $\mathrm{AaDO}_{2}(r=0.47$, $P=0.0021)$ than non-ARDS patients [Table 3].

\section{Discussion}

In this study, we tested the correlation between TPTD parameters (EVLWI and PVPI) and severity of lung injury in terms of oxygenation parameters. We also tried to assess pulmonary edema noninvasively by chest radiograph scoring, ${ }^{[5]}$ and their correlation with EVLWI and PVPI measurements. EVLW was indexed to predicted body weight, instead of the actual body weight, as it has been found to be better reflective of the patient's prognosis. ${ }^{[6-9]}$ Though it is costly and cumbersome to initiate in critical care settings, measuring EVLWI by TPTD gives a repeatable quantitative measure of pulmonary edema that is sensitive to small changes in lung water. ${ }^{[10]}$ Qualitative interpretation of chest radiographs by clinicians is susceptible to inter-observer variability and disagreement. ${ }^{[1]}$ Chest radiograph interpretation is often hindered by positioning of the patients and other conditions such as pleural effusion, consolidation, and atelectasis. Indeed, three chest radiographs were rejected due to the presence of pleural effusion in our study. Our rationale to correlate EVLWI values and chest radiograph scores is that chest radiographs are cheaper and easier to obtain. Previous studies where EVLWI was derived by single dye dilution ${ }^{[5]}$ or thermodilution $\left(\mathrm{PiCCO}^{\circledR}\right.$, Pulsion Medical System, Munich, Germany) technique ${ }^{[11,12]}$ also showed moderate correlation with chest radiograph scores similar to our findings.

The present study showed a moderate negative correlation between EVLWI and $\mathrm{PaO}_{2}: \mathrm{FiO}_{2}$ ratio. This finding was similar to the results of several previous studies. ${ }^{[12,13]}$ We found a moderate correlation between EVLWI and $\mathrm{AaDO}_{2}$ as found in earlier studies ${ }^{[14,15]}$ but in contradiction with the findings of a study ${ }^{[16]}$ where double indicator dilution system was used.

Table 2: Correlation coefficients and $P$ values of correlation

\begin{tabular}{lllll}
\hline & & No. of values $(\boldsymbol{n})$ & Correlation coefficient $(\boldsymbol{r})$ \\
\hline EVLWI & Chest radiograph score (Observer 1) & 64 & 0.71 & $<$ \\
EVLWI & Chest radiograph score (Observer 2) & 64 & 0.68 & $<0.0001$ \\
EVLWI & $\mathrm{PaO}_{2} / \mathrm{FiO}_{2}$ & 117 & -0.32 & 0.0001 \\
EVLWI & $\mathrm{AaDO}_{2}$ & 117 & 0.29 & 0.0004 \\
EVLWI & $\mathrm{PVPI}$ & 99 & 0.88 & $<0.0001$ \\
PVPI & $\mathrm{Chest} \mathrm{radiograph} \mathrm{score} \mathrm{(Observer} \mathrm{1)}$ & 57 & 0.60 & $<0.0001$ \\
PVPI & $\mathrm{Chest} \mathrm{radiograph} \mathrm{score} \mathrm{(Observer} \mathrm{2)}_{2}$ & 57 & 0.59 & $<0.0001$ \\
PVPI & $\mathrm{PaO}_{2} / \mathrm{FiO}_{2}$ & 99 & -0.39 & 0.0001 \\
PVPI & $\mathrm{AaDO}_{2}$ & 99 & 0.32 & 0.0013 \\
\hline
\end{tabular}

EVLWI: Extravascular lung water index; PVPI: Pulmonary Vascular Permeability Index 


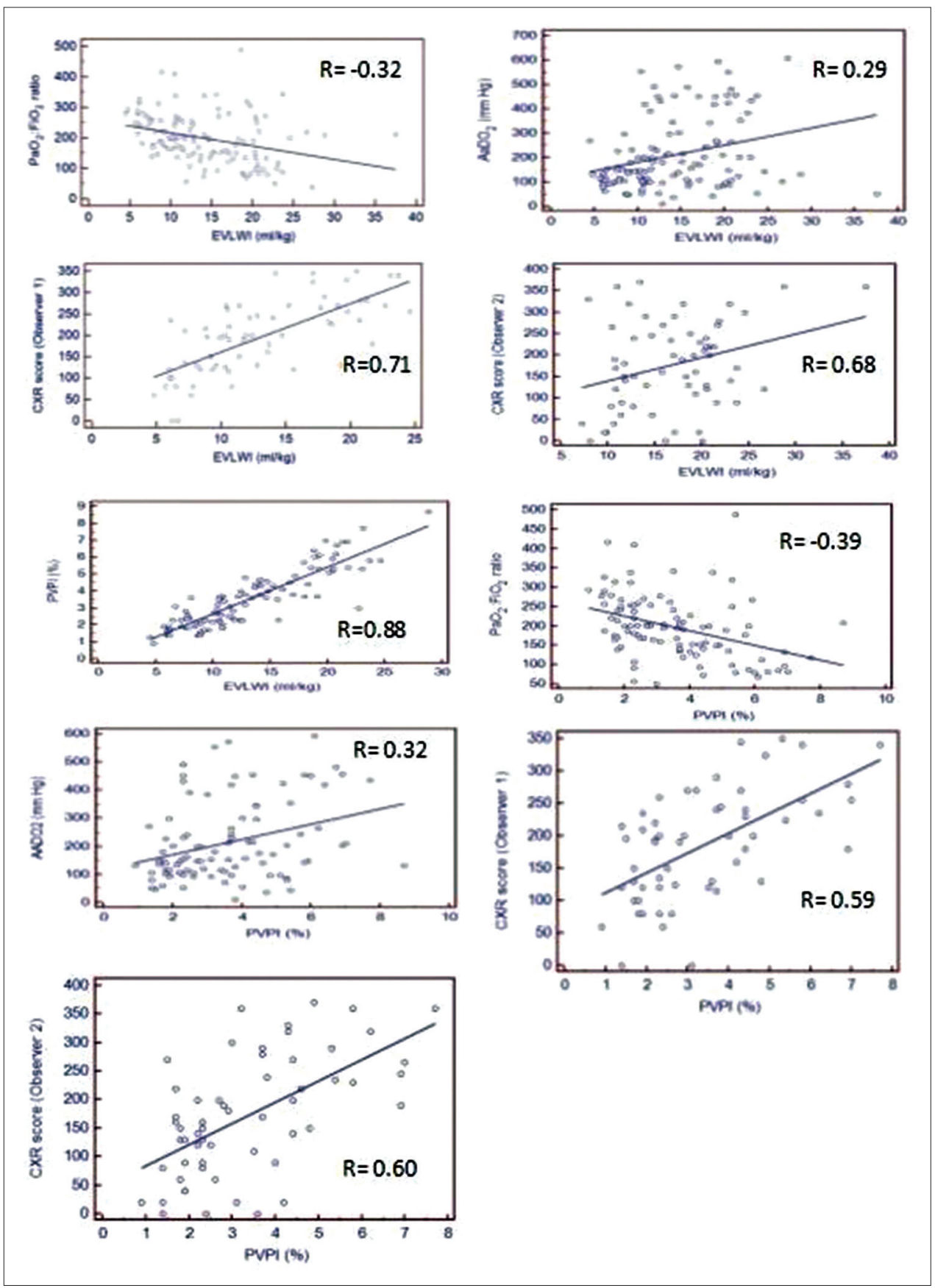

Figure 1: Scatter plots showing correlation between extravascular lung water index, pulmonary vascular permeability index, chest radiograph scores, $\mathrm{PaO}_{2}: \mathrm{FiO}_{2}$ ratio and Alveolo-arterial gradient of oxygen tension

Increases in EVLWI and PVPI are the indicators of common physiological derangements in septic shock and ARDS due to increased capillary leakiness which allows protein-rich fluid to escape through the capillary endothelium. ${ }^{[17]}$ PVPI is calculated as the ratio of EVLWI and PBV. This may explain the strong correlation between PVPI and EVLWI in our study. Clinical studies have shown significantly higher PVPI in ARDS than in hydrostatic pulmonary edema. ${ }^{[18]}$ An increase in pulmonary vascular permeability leads to increased EVLW and hence decreased lung compliance. Alveolar flooding due to increased permeability causes intrapulmonary shunt-related hypoxemia.
However, we found only a moderate correlation of PVPI with $\mathrm{PaO}_{2}: \mathrm{FiO}_{2}$ ratio and $\mathrm{AaDO}_{2}$.

In the present study, baseline EVLWI and PVPI were higher among ARDS patients than non-ARDS patients, but it was not statistically significant. This finding can be attributed to the confounding effect of sepsis and multiorgan dysfunction on the permeability and extravascular water content as all of our patients were in septic shock with multiorgan dysfunction. Indeed, in a previous study by Martin et al., 27\% of patients with clinical ARDS never had raised EVLWI and 57\% of 


\begin{tabular}{|c|c|c|c|}
\hline & ARDS patients $(N=9)$ & Non-ARDS patients $(N=11)$ & $P$ \\
\hline Baseline EVLWI (ml/kg) & $14.7778 \pm 6.2229$ & $12.1818 \pm 6.2199$ & 0.3655 \\
\hline Baseline PVPI (\%) & $4.0667 \pm 2.1354$ & $3.4727 \pm 1.5825$ & 0.4839 \\
\hline Correlation between EVLWI and $\mathrm{PaO}_{2}: \mathrm{FiO}_{2}$ & $n=44, \mathrm{r}=-0.71(P<0.0001)$ & $n=73, \mathrm{r}=-0.24(P=0.0419)$ & 0.0011 \\
\hline Correlation between EVLWI and $\mathrm{AaDO}_{2}$ & $n=44, \mathrm{r}=0.66(P<0.0001)$ & $n=73, \mathrm{r}=0.14(P=0.2430)$ & 0.0009 \\
\hline Correlation between PVPI and $\mathrm{PaO}_{2}: \mathrm{FiO}_{2}$ & $n=40, \mathrm{r}=-0.58(P=0.0001)$ & $n=59, \mathrm{r}=-0.26(P=0.0468)$ & 0.0614 \\
\hline Correlation between PVPI and $\mathrm{AaDO}_{2}$ & $n=40, \mathrm{r}=0.47(P=0.0021)$ & $n=59, \mathrm{r}=0.19(P=0.1413)$ & 0.1337 \\
\hline
\end{tabular}

Baseline EVLWI and PVPI are expressed as Mean \pm Standard deviation, $N$ : Number of patients; $n$ : Number of observations; r: Correlation coefficient; EVLWI: Extravascular lung water index; PVPI: Pulmonary vascular permeability index

patients with severe sepsis had raised EVLWI in the absence of clinical ARDS, suggesting an unrecognized form of lung injury as a part of multiorgan dysfunction that does not fulfill the Berlin definition. ${ }^{[12]}$ This can be further supported by the findings of a previous study where EVLWI and PVPI were higher in patients with sepsis-induced multiorgan dysfunction syndrome than patients without. Statistically significant higher values were obtained on both days 1 and 3 in sepsis of pulmonary and nonpulmonary origin, indicating the role of sepsis-induced increased permeability with or without ARDS. ${ }^{[19]}$

In the subgroup analysis of patients with ARDS, we found a better correlation of EVLWI and PVPI with $\mathrm{PaO}_{2}: \mathrm{FiO}_{2}$ and $\mathrm{AaDO}_{2}$. This is a strong reflection of the innate pathophysiology of ARDS, where intrapulmonary shunting is a major contributor of hypoxemia as explained earlier. Whereas in non-ARDS patients along with sepsis-induced increased permeability, there are many other causes of hypoxemia including ventilation-perfusion mismatch. The above finding does not agree with the hypothesis of the negative effect of dead space ventilation, which is a part of ARDS pathophysiology, on the validity of TPTD parameters, but further studies with higher number of patients are needed to strengthen this fact. In an earlier study, Phillips et al. ${ }^{[9]}$ reported lack of decrease in EVLW indexed to PBW (EVLW $\left.{ }_{\mathrm{p}}\right)$ at maximum values of dead space ventilation.

Indices of oxygenation and EVLWI are independent predictors of ARDS with their own physiological importance. PVPI and EVLWI as independent predictors of mortality indicate different pathogenesis of ARDS. While PVPI quantifies the alveolocapillary barrier permeability, EVLWI measures the impact of this on pulmonary capillary leak. ${ }^{[2]}$ Earlier studies had found a good correlation between PVPI and prognosis of ARDS patients and it was established as an independent mortality indicator ${ }^{[18]}$ In the present study, the base line EVLWI and PVPI were not different significantly. In 200 ARDS patients, Jozwiak et al.$^{[18]}$ had reported a poor predictive value of EVLWI on day-1 compared to the value on day-3 and EVLWI value reached maximum within 3 days on average. Similar to above findings, we had a good correlation between TPTD parameters and oxygenation indices among ARDS patients when all the measurements were taken into account.
Our study has some limitations. First, the sample size was relatively small. However, the measurements were done at several points on each patient to provide adequate power. This could have led to bias which can be prevented by a larger sample size. Second, there may be concerns regarding the reliability of TPTD in severe ventilation-perfusion mismatch hampering access to the poorly perfused pulmonary vascular bed. ${ }^{[20]}$ Third, even though we excluded three chest radiographs due to evident pleural effusion, we could not use ultrasound or CT scan to rule out minimal pleural effusion. Fourth, due to small study population, we could not assess the prognostic value of EVLWI in terms of mortality outcomes and the impact of negative fluid balance aiming at reduction of EVLWI on oxygenation and other physiologic variables. Lastly, fluid balance could not be recorded in a protocolized manner due to increased physician and nursing workload.

\section{Conclusions}

Even though we have found a modest correlation of TPTD parameters with oxygenation, the correlation was significant in the subgroup of patients with ARDS. Thus, we conclude that EVLWI and PVPI may have a prognostic significance in the assessment of lung injury in septic shock patients with ARDS, and targeting fluid management towards decreasing EVLWI may improve oxygenation in patients with ARDS. Further studies with larger sample size are required to confirm these findings and to reveal the usefulness of EVLWI and PVPI as end points of fluid resuscitation in the management of septic shock with ARDS.

\section{Acknowledgment}

Department of Biotechnology, Ministry of Science and Technology for financial support for the conduct of the research work.

\section{Financial support and sponsorship}

Department of Biotechnology, Ministry of Science and Technology, Government of India.

\section{Conflicts of interest}

There are no conflicts of interest.

\section{REFERENCES}

1. Perel A, Monnet X. Extravascular lung water. In: Vincent J, Hall J, editos. Encyclopedia of Intensive Care Medicine. Berlin Heidelberg: Springer-Verlag; 2011. 
2. Jozwiak M, Teboul JL, Monnet X. Extravascular lung water in critical care: Recent advances and clinical applications. Ann Intensive Care 2015;5:38.

3. Practice parameters for hemodynamic support of sepsis in adult patients in sepsis. Task Force of the American College of Critical Care Medicine, Society of Critical Care Medicine. Crit Care Med 1999;27:639-60.

4. Sakka SG, Klein M, Reinhart K, Meier-Hellmann A. Prognostic value of extravascular lung water in critically ill patients. Chest 2002;122:2080-6.

5. Halperin BD, Feeley TW, Mihm FG, Chiles C, Guthaner DF, Blank NE, et al. Evaluation of the portable chest roentgenogram for quantitating extravascular lung water in critically ill adults. Chest 1985;88:649-52.

6. Chew MS, Ihrman L, During J, Bergenzaun L, Ersson A, Undén J, et al. Extravascular lung water index improves the diagnostic accuracy of lung injury in patients with shock. Crit Care 2012;16:R1.

7. Berkowitz DM, Danai PA, Eaton S, Moss M, Martin GS. Accurate characterization of extravascular lung water in acute respiratory distress syndrome. Crit Care Med 2008;36:1803-9.

8. Craig TR, Duffy MJ, Shyamsundar M, McDowell C, McLaughlin B, Elborn JS, et al. Extravascular lung water indexed to predicted body weight is a novel predictor of Intensive Care Unit mortality in patients with acute lung injury. Crit Care Med 2010;38:114-20.

9. Phillips CR, Chesnutt MS, Smith SM. Extravascular lung water in sepsis-associated acute respiratory distress syndrome: Indexing with predicted body weight improves correlation with severity of illness and survival. Crit Care Med 2008;36:69-73.

10. Fernández-Mondéjar E, Rivera-Fernández R, García-Delgado $M$, Touma A, Machado J, Chavero J, et al. Small increases in extravascular lung water are accurately detected by transpulmonary thermodilution. J Trauma 2005;59:1420-3.

11. Brown LM, Calfee CS, Howard JP, Craig TR, Matthay MA, McAuley DF, et al. Comparison of thermodilution measured extravascular lung water with chest radiographic assessment of pulmonary oedema in patients with acute lung injury. Ann Intensive Care 2013;3:25.

12. Martin GS, Eaton S, Mealer M, Moss M. Extravascular lung water in patients with severe sepsis: A prospective cohort study. Crit Care 2005;9:R74-82.

13. Szakmany T, Heigl P, Molnar Z. Correlation between extravascular lung water and oxygenation in ALI/ARDS patients in septic shock: Possible role in the development of atelectasis? Anaesth Intensive Care 2004;32:196-201

14. Laggner A, Kleinberger G, Sommer G, Haller J, Lenz K, Base W, et al. Determination of extravascular lung water in critical patients: Comparison with radiological, hemodynamic and functional lung findings. Schweiz Med Wochenschr 1985;115:210-3.

15. Touho H, Karasawa J, Shishido H, Yamada K, Yamazaki Y. Hypoxemia in the acute stage of hypertensive intracerebral hemorrhage, with special reference to increased extravascular lung water. Neurol Med Chir (Tokyo) 1989;29:724-7.

16. Knoch M, Vogell H, Höltermann W, Müller E, Lennartz H. The measurement of extravascular lung water - Significant in the follow-up of ARDS? Anasth Intensivther Notfallmed 1990;25:411-5.

17. Tyagi A, Sethi AK, Girotra G, Mohta M. The microcirculation in sepsis. Indian J Anaesth 2009;53:281-93.

18. Jozwiak M, Silva S, Persichini R, Anguel N, Osman D, Richard C, et al. Extravascular lung water is an independent prognostic factor in patients with acute respiratory distress syndrome. Crit Care Med 2013;41:472-80.

19. Chung FT, Lin HC, Kuo CH, Yu CT, Chou CL, Lee KY, et al. Extravascular lung water correlates multiorgan dysfunction syndrome and mortality in sepsis. PLoS One 2010;5:e15265.

20. Matthay MA. Clinical measurement of pulmonary edema. Chest 2002;122:1877-9. 